\title{
Characteristics of COVID-19 at a non-COVID tertiary pulmonary care centre in Delhi, India
}

\author{
Nitin Goel$^{1}$, Sonam Spalgais ${ }^{1}$, Parul Mrigpuri ${ }^{1}$, Madhu Khanna ${ }^{2}$, Balakrishnan Menon', Raj Kumar ${ }^{1}$ \\ ${ }^{1}$ Department of Pulmonary Medicine, Vallabhbhai Patel Chest Institute, University of Delhi, New Delhi; ${ }^{2}$ Virology \\ Unit, Department of Microbiology, Vallabhbhai Patel Chest Institute, University of Delhi, New Delhi, India
}

\begin{abstract}
The pandemic of COVID-19 has emerged as a serious health crisis globally and India too has been extensively affected with 604,641 active cases reported, till date. The present study focuses on the demographic, clinical and laboratory profile of such patients from a tertiary level non-COVID respiratory care hospital. This is a retrospective observational study. Seventy-seven sick patients fulfilling COVID suspect criteria were admitted to the isolation area. Their RT-PCR test was done from the designated laboratory and 35 of them were confirmed to be COVID-19 patients. The detailed demographic, clinical and laboratory profile of these COVID-19 patients was studied. The mean age was
\end{abstract}

\section{Correspondence: Raj Kumar, Department of Pulmonary Medicine, Vallabhbhai Patel Chest Institute, University of Delhi, New Delhi 110007 , India. \\ Tel. +91.9810146835; 91-011-27667667 Ext: 109. \\ E-mail: rajkumarvpci@gmail.com}

Key words: COVID-19; lung diseases; comorbidity; pandemic; Severe Acute Respiratory Syndrome Coronavirus 2.

Authors' contributions: NG, BM, conception and design, drafting the article, final approval of the version; SS, PM, MK, analysis and interpretation, drafting the article, final approval of the version; RK, conception and design, analysis and interpretation, drafting the article, revising it critically, final approval of the version and agreement to be accountable for all aspects of the work.

Conflict of interest: The authors declare no conflict of interest.

Ethics approval: The present study was approved by the Institutional Human Ethics Committee (IHEC) vide number VPCI/ADMN. II/JRO/IHEC/2020/708, dated July $22^{\text {nd }}, 2020$.

Informed consent: Obtained from participants.

Received for publication: 12 August 2020.

Accepted for publication: 13 September 2020.

${ }^{\circ}$ Copyright: the Author(s), 2020

Licensee PAGEPress, Italy

Monaldi Archives for Chest Disease 2020; 90:1568

doi: 10.4081/monaldi.2020.1568

This article is distributed under the terms of the Creative Commons Attribution Noncommercial License (by-nc 4.0) which permits any noncommercial use, distribution, and reproduction in any medium, provided the original author(s) and source are credited.
$46 \pm 17$ years with male predominance (57\%). Majority of the cases $(83 \%)$ were symptomatic. The most common symptom was cough (66\%) followed by breathlessness and fever. Nineteen $(54.3 \%)$ patients had one or the other co-morbidity and $16(45.7 \%)$ had chronic lung diseases as one of the comorbidities. Nearly half of the patients $(51 \%)$ required supplementary oxygen on presentation. Two patients were put on invasive mechanical ventilation while 4 patients required non-invasive ventilation before being shifted to the COVID hospital. Hence, it can be concluded that COVID-19 in patients of chronic respiratory diseases manifests with higher prevalence of symptoms and also higher severity of disease. Further, the symptomatology of COVID-19 closely mimics the acute exacerbation of chronic lung diseases, so cautious screening and testing should be done, especially at the pulmonary department.

\section{Introduction}

The novel corona outbreak emerged in the Wuhan province of China in December 2019 and rapidly spread all over the world. Subsequently, it was declared as public health emergency of international concern by World Health Organization [1]. As on $2^{\text {nd }}$ July 2020, there were 604,641 active cases in India with 17,834 deaths [2]. Coronaviruses are known to cause respiratory diseases and have been responsible for severe acute respiratory syndrome (SARS) and middle eastern respiratory syndrome (MERS) outbreaks in 2003 and 2012, respectively [3,4]. Epidemiological, clinical and laboratory profile of COVID-19 patients has been discussed by various studies from China [5,6]. Recently studies focussing on the clinical profile of the initial COVID-19 patients, managed in designated COVID hospitals, from India have also been published [7,8]. Our study primarily focuses on the demographic, clinical and laboratory profile of COVID-19 patients presenting to a tertiary level nonCOVID respiratory care hospital in Delhi.

\section{Materials and Methods}

This is a retrospective observational study and was carried out in a tertiary care centre dedicated to the management of respiratory diseases. In the present study, we analysed the epidemiological and clinical features of patients diagnosed to be suffering from COVID 19 in our set up. As per the Ministry of Health and Family Welfare (MOHFW) of the Government of India, some hospitals were designated COVID hospitals for the management of COVID-19 patients [9]. Our hospital however is a non-COVID 
hospital and in the present situation catering to the patients of other respiratory diseases like COPD, bronchial asthma, tuberculosis, interstitial lung diseases (ILD), bronchiectasis, etc.

A screening centre has been set up at the entry point of our hospital for screening the patients with history or symptoms suggestive of COVID-19. A screening proforma is also filled for all the patients visiting the hospital. The proforma comprises of questions on the history and symptoms suggestive of COVID-19 as well as the COVID-19 suspect criteria as per Indian Council of Medical Research (ICMR) [10]. Also, all the Health Care Workers (HCWs) in our hospital strictly follow the protocol of personal protective equipment (PPE) use as per the recommendations of MOHFW of the Government of India [11].

Further all sick patients fulfilling COVID-19 suspect criteria are admitted to a designated isolation area. Their nasopharyngeal and oropharyngeal swabs are tested at the Department of Microbiology (Virology Unit) of our institute for SARS-CoV-2. In the present study, we describe the epidemiologic and clinical profile of 35 such patients who were confirmed to be positive for SARS-CoV-2.

\section{Results}

On screening the patients as per the ICMR criteria [10], we found 77 patients fulfilling the COVID-19 suspect criteria over a period of time from 08-05-2020 to 03-07-2020. Out of these 77 suspected COVID-19 cases, 35 patients were confirmed to be COVID-19 positive by RT-PCR. The demographic profile of these patients is shown in Table 1.

Severe acute respiratory infection (SARI) was the presenting feature in $22(62.8 \%)$ patients. Seven $(20 \%)$ of the COVID-19 patients were symptomatic employees/health care workers (HCWs) while $3(8.6 \%)$ were HCWs with breach in PPE. There were three cases $(8.6 \%)$ with history of contact with confirmed positive patients. The clinical characteristics and investigations of the patients are shown in Table 2. Most of the patients $29(82.9 \%)$ were symptomatic with mean duration of symptoms being 5.4 days before presenting to the health care facility. The most common symptom was cough which was present in $23(65.7 \%)$ patients followed by breathlessness (60\%) and fever (45.7\%). Five $(14.9 \%)$ patients reported sore throat, two $(5.7 \%)$ patients had diarrhoea and only $1(2.9 \%)$ had headache. Out of the 35 COVID-19 patients, $19(54.3 \%)$ patients had at least one co-morbidity. Further $16(45.7 \%)$ had associated chronic lung disease(s) as the comorbid condition. The majority of patients had multiple co-morbidities $(n=13,68.4 \%)$ and the remaining had single co-morbidity $(n=6$, $31.65 \%)$. Also, $4(11.42 \%)$ patients had diabetes mellitus with chronic lung disease and $3(8.57 \%)$ patients had hypertension with chronic lung disease as the associated comorbidities. Table 3 shows the details of associated co-morbid conditions. Nearly half $(51.4 \%)$ of the patients required oxygen on admission. Two $(5.7 \%)$ patients had to be put on invasive mechanical ventilation while 4 (11.4\%) patients required non-invasive ventilation before being shifted to the COVID hospital.

\section{Discussion}

The COVID-19 disease has affected the whole world with nearly 1 crore confirmed cases and more than 0.5 million deaths
Table 1. Demographic profile of the patients.

\begin{tabular}{lc} 
Characteristic & n (total $n=35)$ \\
Age $($ mean \pm SD) & $46 \pm 17$ years \\
Gender & \\
Male & $20(57.1 \%)$ \\
Female & $15(42.9 \%)$ \\
\hline Nationality & $\mathrm{Nil}$ \\
Foreign & $35(100 \%)$ \\
Indian &
\end{tabular}

Table 2. Clinical characteristics and investigations of the patients.

\begin{tabular}{|c|c|}
\hline Clinical characteristic & n (\%) (total $\mathrm{n}=35)$ \\
\hline \multicolumn{2}{|l|}{ Symptoms } \\
\hline Symptomatic & $29(82.9 \%)$ \\
\hline Asymptomatic & $6(17.1 \%)$ \\
\hline Pulse (mean \pm SD) & $93.5 \pm 15.02 / \mathrm{min}$ \\
\hline Respiratory rate (mean \pm SD) & $15 \pm 2.09 / \mathrm{min}$ \\
\hline \multicolumn{2}{|l|}{$\mathrm{SPO}_{2}$} \\
\hline Normal & $17(48.6 \%)$ \\
\hline Hypoxic & $12(34.3 \%)$ \\
\hline On domiciliary oxygen therapy & $6(17.1 \%)$ \\
\hline \multicolumn{2}{|l|}{ Investigations } \\
\hline $\mathrm{PaO}_{2}($ mean $\pm \mathrm{SD})$ & $69.40 \pm 17.55 \mathrm{mmHg}$ \\
\hline $\mathrm{PaCO}_{2}($ mean $\pm \mathrm{SD})$ & $39.77 \pm 5.49 \mathrm{mmHg}$ \\
\hline $\mathrm{PaO}_{2} / \mathrm{FiO}_{2}($ mean $\pm \mathrm{SD})$ & $316.80 \pm 97.93$ \\
\hline Haemoglobin (mean \pm SD) & $11.9 \pm 1.97 \mathrm{~g} / \mathrm{dl}$ \\
\hline Total leukocyte count (TLC) (mean \pm SD) & $8837.9 \pm 4178.9 / \mathrm{cu} \mathrm{mm}$ \\
\hline Leukocytosis (TLC > 12000/mm3) & $6(17.1 \%)$ \\
\hline Leukopenia (TLC < 4,000/ mm3) & $2(5.7 \%)$ \\
\hline \multicolumn{2}{|l|}{ Kidney function test } \\
\hline Normal & $31(88.6 \%)$ \\
\hline Deranged & $4(11.4 \%)$ \\
\hline \multicolumn{2}{|l|}{ Liver function test } \\
\hline Normal & $31(88.6 \%)$ \\
\hline Deranged & $4(11.4 \%)$ \\
\hline
\end{tabular}

Table 3. Associated comorbidities of the COVID-19 patients $(\mathbf{n}=35)$.

\begin{tabular}{lc} 
Co-morbidities $(\mathrm{n}=19)$ & $\mathrm{n}(\%) *$ \\
Bronchial asthma & $4(21.1 \%)$ \\
Chronic obstructive pulmonary disease & $3(15.8 \%)$ \\
\hline Interstitial lung diseases & $3(15.8 \%)$ \\
Bronchiectasis & $1(5.3)$ \\
\hline Post tubercular sequela & $1(5.3)$ \\
Diabetes mellitus & $2(10.5 \%)$ \\
\hline Hypertension with diabetes mellitus & $1(5.3)$ \\
COPD with post tubercular sequela & $2(10.5 \%)$ \\
\hline ILD with post tubercular sequela & $1(5.3)$ \\
BA with bronchiectasis & $1(5.3)$ \\
\hline
\end{tabular}

*Percentages are out of $n=19$. BA, bronchial asthma; COPD, chronic obstructive airway disease; ILD, interstitial lung diseases. 
till date. India has also been immensely affected with $>0.6$ million confirmed cases and more than 17,000 deaths reported till now [2]. The worst affected states being Maharashtra, Delhi, and Tamil Nadu. PPE including masks or respirators are the first line of defence against this spread of SARS-CoV-2 infection [12]. Hence their appropriate use is paramount for controlling the increase in incidence of COVID-19, especially in hospital settings. In the present study, we found 77 patients fulfilling the suspect criteria for COVID-19 [10] and out of these 35 patients were confirmed to be positive by RT-PCR. The mean age of these COVID-19 positive patients was $46 \pm 17$ years. Similar to our study, the middle age predominance has been seen in various previous national and international studies $[7,813]$. The present series comprised of $22(62.8 \%)$ patients who presented to our hospital emergency, 10 health care workers and 3 cases with history of contact with confirmed COVID-19 positive patients. None of the patients had history of international travel. The epidemiology of our cases is different from the previously reported series from India [7,8]. This difference in epidemiological profile may be due to the current stage of infection in India and especially in Delhi, where the number of hotspots/containment areas have rapidly increased. Early in the second stage of infection it was limited to foreigners, foreign travellers, and contacts of confirmed cases. On detailed history, it was suspected that probably local residential contacts had a major role to play in the COVID-19 positivity of employees/health care workers in the present series.

The most common symptom in our study patients was cough $(66 \%)$, followed by breathlessness (60\%) and fever $(45.7 \%)$. Nearly half of patients $(51 \%)$ presented with hypoxemia and oxygen requirement. Similar symptoms have also been reported from various national and international studies of COVID-19 infection $[5,7,8,13]$. The majority of cases $29(83 \%)$ were symptomatic and 19 (54\%) of patients had at least one comorbidity (Table 3). Overall, $16(45.7 \%)$ patients had chronic lung diseases as co-morbid condition. The majority of patients had multiple co-morbidities $(n=13,68.4 \%)$. Chronic lung diseases $(n=16,45.7 \%)$ were the most common associated comorbidity followed by diabetes mellitus and hypertension. Both chronic lung disease and diabetes mellitus were present together in $11.42 \%(n=4)$ of the patients whereas hypertension with chronic lung disease were the associated comorbidities in $8.57 \%(n=3)$. Ten patients presented with very severe disease, two cases required mechanical ventilation and 4 cases were managed with non-invasive ventilation before being shifted to the designated COVID hospitals. Nearly half of patients $(51 \%)$ required supplementary oxygen. Also, it cannot be overemphasized that all these procedures viz. oxygen therapy, intubation and non-invasive ventilation are aerosol generating procedures and all involved HCWs have to follow stringent PPE guidelines while performing these [14]. Hence, the HCWs in our set up always follow the guidelines on the appropriate use of PPE [11]. We observed higher number of symptomatic patients and severity of disease varying from moderate to severe, with higher number of hypoxemic patients in the present cohort compared to other studies. This may be due to underlying chronic lung diseases (45.7\%) in the present study cohort and also because of the delay in presentation to the health care facility. Since, our institute is a dedicated respiratory diseases institute, nearly $45 \%$ of the patients had at least one associated respiratory co-morbidity. Also, the mean duration of symptoms before presentation was 5.4 days.

Similarities of clinical features between COVID-19 and previous coronavirus infections have been noted. The features of COVID-19 infection have close resemblance to severe acute respiratory syndrome coronavirus (SARS-CoV) and Middle East respi- ratory syndrome coronavirus (MERS-CoV) infections. However, it has been observed that patients with the COVID-19 infection infrequently develop intestinal symptoms whereas as the patients with MERS-CoV or SARS-CoV infection had diarrhoea in about $20-25 \%[15,16]$. Although a recent meta-analysis of 2477 confirmed COVID -19 patients found that the gastrointestinal symptoms are an important clinical feature of COVID-19 (with diarrhea being the most common, present in $7.8 \%$ cases), it is still much less than the prevalence of gastrointestinal symptoms seen in other coronavirus infections [17]. The symptoms of COVID-19 mimic the symptoms of acute exacerbation of chronic lung diseases like COPD and asthma. This poses a challenge for early recognition and diagnosis of COVID-19 infection particularly at a pulmonary centre/department.

Overall, there is lack of data of COVID-19 infection in COPD patients. However, Lippi G and Henry BM [18] in a meta-analysis of 1592 COVID-19 patients reported that COPD was significantly associated with severe COVID-19 (OR: 5.69, 95CI:2.49-13.00). They also demonstrated that COPD is associated with a significant, over five-fold increased risk of severe COVID-19 infection. One of the significant reasons found for this is the occurrence of increased expression of angiotensin converting enzyme -2 (ACE2) receptor by bronchial epithelial cells of patients with COPD. This ACE-2 receptor helps in the entry of SARS-CoV-2 into the cell [19]. Similarly, there is very limited data of COVID-19 infection in patients of asthma. Garg et al. [20] reported that chronic lung disease (primarily asthma) is one of the commonly associated conditions and asthma may increase the risk of hospitalization from COVID-19 in 18-49-year adults. With the increase in COVID-19 infection, the suspected cases will also increase in nonCOVID hospitals particularly in the pulmonary outpatient department (OPD) and the emergency. The diagnosis of COVID-19 in this setting is also more difficult as most of the symptoms are similar to acute exacerbation of chronic lung diseases. Hence, there should be a dedicated screening and testing facility at every pulmonary department for early detection of the COVID-19 and also to prevent further spread of this highly infectious disease amongst chronic respiratory disease patients.

\section{Conclusions}

With the rise in COVID-19 patients in the country, increased number of suspected cases are presenting to non-COVID hospitals with more symptoms and higher severity of the disease. The exacerbations of chronic respiratory disease closely mimic COVID-19 and is also one of the common co-morbidities associated with COVID-19. Hence, every pulmonary department/hospital should have dedicated screening and testing facility for COVID-19 infection to prevent cross infection of the other respiratory disease patients.

\section{References}

1. WHO. Statement on the second meeting of the International Health Regulations (2005) Emergency Committee regarding the outbreak of novel coronavirus (2019-nCoV). Accessed on 2020 Jun 26. Available from: https://www.who.int/newsroom/detail/30-01-2020-statement-on-the-second-meeting-ofthe-international-health-regulations-(2005)-emergency-commit- 
tee-regarding-the-outbreak-of-novel-coronavirus-(2019-ncov)

2. WHO. Coronavirus Disease (COVID-19) Dashboard. Accessed on: 2020 June 25. Available from: https://covid19.who.int/

3. Chatterjee P, Nagi N, Agarwal A, et al. The 2019 novel coronavirus disease (COVID-19) pandemic: A review of the current evidence. Indian J Med Res2020;151:147.

4. Adhikari SP, Meng S, Wu Y-J, et al. Epidemiology, causes, clinical manifestation and diagnosis, prevention and control of coronavirus disease (COVID-19) during the early outbreak period: a scoping review. Infect Dis Poverty 2020;9:29.

5. Huang C, Wang Y, Li X, et al. Clinical features of patients infected with 2019 novel coronavirus in Wuhan, China. Lancet 2020;395:497-506.

6. Wang D, Hu B, Hu C, et al. Clinical characteristics of 138 hospitalized patients with 2019 novel Coronavirus-infected pneumonia in Wuhan, China. JAMA 2020;323:1061-9.

7. Gupta N, Agrawal S, Ish P, et al. Clinical and epidemiologic profile of the initial COVID-19 patients at a tertiary care centre in India. Monaldi Arch Chest Dis 2020;90:1294. doi: 10.4081/monaldi.2020.1294

8. Bhandari S, Shaktawat AS, Sharma R, et al. A preliminary clinico-epidemiological portrayal of COVID-19 pandemic at a premier medical institution of North India. Ann Thorac Med 2020;15:146-50.

9. Ministry of Health \& Family Welfare, Government of India. Guidance document on appropriate management of suspect/confirmed cases of COVID-19. Accessed on: 2020 Jul 4. Available from: https://www.mohfw.gov.in/pdf/FinalGuidanceonMangae mentofCOVIDcasesversion2.pdf

10. Indian Council of Medical Research. Strategy for COVID19 testing in India. Accessed on: 2020 Jul 3. Available from: https://www.mohfw.gov.in/pdf/Revisedtestingguidelines.pdf

11. Government of National Capital Territory of Delhi, Health \& Family Welfare Department. Accessed on: 2020 Jul 4. Available from: http://health.delhigovt.nic.in/wps/wcm/connect/222c 64804e1e41a8bfb8ffd194e333e1/GOVT+HOSP.pdf?MOD=AJ PERES\&lmod $=-826499128 \&$ CACHEID $=222 \mathrm{c} 64804 \mathrm{e} 1 \mathrm{e} 41 \mathrm{a} 8$ bfb8ffd194e333e1

12. Ippolito M, Vitale F, Accurso G, et al. Medical masks and respirators for the protection of healthcare workers from SARSCoV-2 and other viruses. Pulmonol 2020;26:204-12

13. Guan W, Ni ZY, Hu Y, et al. Clinical characteristics of coronavirus disease 2019 in China. N Engl J Med 2020;382:1708-20.

14. Winck JC, Ambrosino N. COVID-19 pandemic and non invasive respiratory management: every goliath needs a David. An evidence based evaluation of problems. Pulmonol 2020;26:213-220

15. Lee N, Hui D, Wu A, et al. A major outbreak of severe acute respiratory syndrome in Hong Kong. N Engl J Med 2003;348: 1986-94.

16. Assiri A, Al-Tawfiq JA, Al-Rabeeah AA, et al. Epidemiological, demographic, and clinical characteristics of 47 cases of Middle East respiratory syndrome coronavirus disease from Saudi Arabia: a descriptive study. Lancet Infect Dis 2013;13:752-61.

17. Kumar VCS, Mukherjee S, Harne PS, et al. Novelty in the gut: a systematic review and meta-analysis of the gastrointestinal manifestations of COVID-19. BMJ Open Gastroenterol 2020;7:e000417.

18. Lippi G, Henry BM. Chronic obstructive pulmonary disease is associated with severe coronavirus disease 2019 (COVID-19). Respirat Med 2020;167:105941.

19. Leung JM, Niikura M, Yang CWT, et al. COVID-19 and COPD. Eur Respir J 2020;56:2002108.

20. Garg S, Kim L, Whitaker M, et al. Hospitalization rates and characteristics of patients hospitalized with laboratory-confirmed Coronavirus disease 2019- COVID-NET, 14 States, March 1-30, 2020. MMWR Morb Mortal Wkly Rep 2020;69:458-464. doi: 10.15585/mmwr.mm6915e3 